\title{
Growth and Approximation of Laplace-Stieltjes Transform with $(p, q)$-Proximate Order Converges on the Whole Plane
}

\author{
Yong Qin Cui ${ }^{1}$ and Hong Yan $\mathrm{Xu} \mathbb{i}^{1,2}$ \\ ${ }^{1}$ Department of Informatics and Engineering, Jingdezhen Ceramic Institute, Jingdezhen, Jiangxi 333403, China \\ ${ }^{2}$ School of Mathematics and Computer Science, Shangrao Normal University, Shangrao, Jiangxi 334001, China \\ Correspondence should be addressed to Hong Yan Xu; xhyhhh@126.com
}

Received 24 October 2019; Accepted 10 December 2019; Published 13 February 2020

Academic Editor: Kehe Zhu

Copyright (C) 2020 Yong Qin Cui and Hong Yan Xu. This is an open access article distributed under the Creative Commons Attribution License, which permits unrestricted use, distribution, and reproduction in any medium, provided the original work is properly cited.

\begin{abstract}
One purpose of this paper is to study the growth of entire functions defined by Laplace-Stieltjes transform converges on the whole complex plane, by introducing the concept of $(p, q)$-proximate order, and one equivalence theorem of the $(p, q)$-proximate order of Laplace-Stieltjes transforms is obtained. Besides, the second purpose of this paper is to investigate the approximation of entire functions defined by Laplace-Stieltjes transforms with $(p, q)$-proximate order, and some results about the $(p, q)$-proximate order, the error, and the coefficients of Laplace-Stieltjes transforms are obtained, which are generalization and improvement of the previous theorems given by Luo and Kong, Singhal, and Srivastava.
\end{abstract}

\section{Introduction}

The main purpose of this paper is to investigate the growth and approximation of entire functions represented by Laplace-Stieltjes transforms which convergent on the whole complex plane. For Laplace-Stieltjes transforms,

$$
G(s)=\int_{0}^{+\infty} e^{-s x} \mathrm{~d} \alpha(x), \quad s=\sigma+i t,
$$

where $\alpha(x)$ is a bounded variation on any finite interval $[0, Y](0<Y<+\infty)$ and $\sigma$ and $t$ are real variables. In fact, if $\alpha(t)$ is absolutely continuous, then $G(s)$ becomes the classical Laplace integral form:

$$
G(s)=\int_{0}^{\infty} e^{-s t} \varphi(t) \mathrm{d} t
$$

If $\alpha(t)$ is a step function, we can choose a sequence $\left\{\lambda_{n}\right\}_{0}^{\infty}$ satisfying

$$
0 \leq \lambda_{1}<\lambda_{2}<\cdots<\lambda_{n}<\cdots, \lambda_{n} \longrightarrow \infty, \quad \text { as } n \longrightarrow \infty,
$$

and let

$$
\alpha(x)= \begin{cases}a_{1}+a_{2}+\cdots+a_{n}, & \lambda_{n} \leq x<\lambda_{n+1} \\ 0, & 0 \leq x<\lambda_{1} ; \\ \frac{\alpha(x+)+\alpha(x-)}{2}, & x>0 .\end{cases}
$$

Thus, $G(s)$ becomes a Dirichlet series:

$$
G(s)=\sum_{n=1}^{\infty} a_{n} e^{\lambda_{n} s}, \quad s=\sigma+i t,
$$

where $\sigma$ and $t$ are real variables and $a_{n}$ are nonzero complex numbers; if $\alpha(t)$ is an increasing continuous function which is not absolutely continuous, then integral (1) defines a class of functions $G(s)$ which cannot be expressed either in form (2) or (6).

As we know, the Laplace-Stieltjes transform, named for Pierre-Simon Laplace and Thomas Joannes Stieltjes, is an integral transform similar to the Laplace transform. For realvalued functions, it is the Laplace transform of a Stieltjes 
measure; however, it is often defined for functions with values in a Banach space. It can be used in many fields of mathematics, such as functional analysis, and certain areas of theoretical and applied probability.

In 1963, Yu [1] first proved the Valiron-Knopp-Bohr formula of the associated abscissas of bounded convergence, absolute convergence, and uniform convergence of the LaplaceStieltjes transform.

Theorem 1 (see [1]). If the sequence $\left\{\lambda_{n}\right\}$ satisfy (3) and

$$
\begin{array}{r}
\limsup _{n \longrightarrow+\infty} \frac{\log n}{\lambda_{n}}=D<+\infty, \\
\limsup _{n \longrightarrow+\infty}\left(\lambda_{n+1}-\lambda_{n}\right)=h<+\infty,
\end{array}
$$

then LaplaceStieltjes transforms (1) satisfy

$$
\limsup _{n \longrightarrow+\infty} \frac{\log B_{n}^{*}}{\lambda_{n}} \leq \sigma_{u}^{G} \leq \limsup _{n \longrightarrow+\infty} \frac{\log B_{n}^{*}}{\lambda_{n}}+\operatorname{limup}_{n \longrightarrow+\infty} \frac{\log n}{\lambda_{n}},
$$

where $\sigma_{u}^{G}$ is called the abscissa of uniformly convergent of $F(s)$ and

$$
B_{n}^{*}=\sup _{\lambda_{n}<x \leq \lambda_{n+1},-\infty<t<+\infty}\left|\int_{\lambda_{n}}^{x} e^{-i t y} \mathrm{~d} \alpha(y)\right| .
$$

Moreover, $\mathrm{Yu}$ [1] introduced the concepts of the order of $G(s)$ and estimated the growth of the maximal molecule $M_{u}(\sigma, G)$, the maximal term $\mu(\sigma, G)$, the Borel line, and the order of entire functions represented by LaplaceStieltjes transform convergent in the whole complex plane. After his works, considerable attention has been paid to the growth and the value distribution of the functions represented by LaplaceStieltjes transform convergent in the half plane or whole complex plane in the field of complex analysis (see [2-16]).

In 2012, Luo and Kong [17] studied the following form of the LaplaceStieltjes transform:

$$
F(s)=\int_{0}^{+\infty} e^{s x} \mathrm{~d} \alpha(x), \quad s=\sigma+i t
$$

where $\alpha(x)$ is stated as in (1) and $\left\{\lambda_{n}\right\}$ satisfies (3) and (6), which is a different form from (1). Set

$$
A_{n}^{*}=\sup _{\lambda_{n}<x \leq \lambda_{n+1},-\infty<t<+\infty}\left|\int_{\lambda_{n}}^{x} e^{i t y} \mathrm{~d} \alpha(y)\right| .
$$

By using the same argument as in [1], we can get the similar result about the abscissa of uniformly convergent of $F(s)$ easily. If

$$
\limsup _{n \longrightarrow+\infty} \frac{\log A_{n}^{*}}{\lambda_{n}}=-\infty
$$

by (3), (6), and Theorem 1, we have $\sigma_{u}^{F}=+\infty$; thus, it can be said that $F(s)$ is an entire function in the whole plane.
Set

$$
\begin{aligned}
\mu(\sigma, F) & =\max _{n \in N}\left\{A_{n}^{*} e^{\lambda_{n} \sigma}\right\}(\sigma<+\infty), \\
M(\sigma, F) & =\sup _{-\infty<t<+\infty}|F(\sigma+i t)|, \\
M_{u}(\sigma, F) & =\sup _{0<x<+\infty,-\infty<t<+\infty}\left|\int_{0}^{x} e^{(\sigma+i t) y} \mathrm{~d} \alpha(y)\right| .
\end{aligned}
$$

Since $M(\sigma, F)$ and $M_{u}(\sigma, F)$ trend to $+\infty$ as $\sigma \longrightarrow+\infty$, in order to estimate the growth of $F(s)$ precisely, the concepts of order and type is introduced as follows.

Definition 1. If the LaplaceStieltjes transform (11) satisfies $\sigma_{u}^{F}=+\infty$ (the sequence $\left\{\lambda_{n}\right\}$ satisfy (3), (9), and (20)) and

$$
\limsup _{\sigma \longrightarrow+\infty} \frac{\log ^{+} \log ^{+} M_{u}(\sigma, F)}{\sigma}=\rho, \quad 0 \leq \rho \leq+\infty,
$$

we call $F(s)$ is of order $\rho$ in the whole plane, where $\log ^{+} x=\max \{\log x, 0\}$. Furthermore, if $\rho \in(0,+\infty)$, the type of $F(s)$ is defined by

$$
\underset{\sigma \longrightarrow+\infty}{\limsup } \frac{\log ^{+} M_{u}(\sigma, F)}{e^{\sigma \rho}}=T, \quad 0 \leq T \leq+\infty .
$$

Remark 1. The concept of type is used to compare the growth of two LaplaceStieltjes transforms of the same nonzero finite order. For example, let $f(s)=e^{e^{s}}$ and $g(s)=e^{e^{2 s}}$, by simple computation, and we have $\rho(f)=1=\rho(g)$, but $T(f)=1$ and $T(g)=\infty$. Thus, we can see that the growth of $g(s)$ is faster than $f(s)$ as $|s| \longrightarrow+\infty$.

Remark 2. Valiron [18] used intermediate comparison functions called the proximate order to refine these growth scales, which make it unnecessary to consider functions of minimal or maximal type.

Remark 3. However, if $\rho=0$ and $\rho=+\infty$, we cannot estimate the growth of such functions precisely by using the concept of type.

For $\rho=+\infty$, Kong [19] studied the growth of such functions by using the concepts of the Sun's type function $U(r)=r^{\exp (1 / \rho(r))}$, where $r=e^{-\sigma}, \rho(r)$ is the decreasing function $\downarrow 0, \rho^{\prime}(r) \uparrow$, and $\lim _{r \rightarrow \infty} r \rho^{\prime}(r) \log r \log \log r=0$. In fact, one can also estimate the growth of LaplaceStieltjes transforms by using the concepts of $p$-order were given by Sato [20]. If

$$
\limsup _{\sigma \longrightarrow+\infty} \frac{\log ^{p+1} M_{u}(\sigma, F)}{\sigma}=\rho(p),
$$

where $\log ^{0} x=x, \log \left(\log ^{p} x\right)=\log ^{p+1} x$, and $p \in \mathbb{N}_{+}$, then $F(s)$ is said to be of index $p$ if $\rho(p-1)=\infty$ and $\rho(p)<+\infty$. If $0<\rho(p)<+\infty$, we also compare their growth by using the concepts of $p$-type. However, there are two shortcomings in the concepts of $p$-order and $p$-type as follows. 
S1: one can hardly compare the growth of such functions of zero order by using the idea of $p$-order.

S2: the growth of such functions of $\rho(p-1)=\infty$ and $\rho(p)=0$ is incomparable by using the idea of $p$-order.

For the first shortcoming, there were some concepts such as logarithmic order, $h$-order, and generalize order (see [21-24]). However, to cover these shortcomings simultaneously, the concepts of $(p, q)$-order and $(p, q)$-type were good ideas and were used to estimate the growth of a class of entire functions represented by LaplaceStieltjes transforms more precisely, which were given by Juneja, Kapor, and Bajpai [25-27].

Definition 2. If the LaplaceStieltjes transform (11) satisfies $\sigma_{u}^{F}=+\infty$ (the sequence $\left\{\lambda_{n}\right\}$ satisfy (3), (9), and (20)) and

$$
\rho=\rho(p, q)=\limsup _{\sigma \longrightarrow+\infty} \frac{\log ^{p+1} M_{u}(\sigma, F)}{\log ^{q} \sigma}, \quad 0 \leq \rho(p, q) \leq+\infty,
$$

then we say that $F(s)$ is of $(p, q)$-order $\rho(p, q)$ in the whole plane, where $p, q$ are two integers such that $p \geq q \geq 0$ and $p \neq 0, \quad$ and $\exp ^{0}(x)=\log ^{0}(x)=x, \quad \exp ^{p+1}(x)=\exp$ $\left(\exp ^{p}(x)\right)$, and $\log ^{-1}(x)=\exp (x), \exp ^{-1}(x)=\log x$.

Remark 4. Throughout this paper, the conditions $p \geq q \geq 0$ and $p \neq 0$ are always supposed to hold.

Remark 5. It can be seen that $0 \leq \rho(p, q) \leq+\infty$ if $p>q$ and $1 \leq \rho(p, q) \leq+\infty$ if $p=q$. Furthermore, if $0 \leq \rho(p, q) \leq+\infty$, then $\rho(p-n, q)=\infty$ for $n<p, \rho(p, q-n)=0$ for $n<q$, and $\rho(p+n, q+n)=1$ for $n=1,2, \ldots$

Definition 3. It is said that the LaplaceStieltjes transform $F(s)$ is of index-pair $(p, q), p \geq q \geq 0$, and $p \neq 0$ if $b<$ $\rho(p, q)<\infty$, and $\rho(p-1, q-1)$ is not a finite nonzero number, where $b=1$ if $p=q$ and $b=0$ if $p>q$. If $F(s)$ is of index-pair $(p, q)$, then $\rho \equiv \rho(p, q)$ is called its $(p, q)$-order.

The concept of $(p, q)$-type is introduced below, which is used to compare the growth of such functions of the same $(p, q)$-order.

Definition 4. The LaplaceStieltjes transform (11) of $(p, q)$-order $\rho(p, q) \in(0,+\infty)$ is said to be of the $(p, q)$-type if

$$
T=T(p, q)=\limsup _{\sigma \rightarrow+\infty} \frac{\log ^{p} M_{u}(\sigma, F)}{\left(\log ^{q-1} \sigma\right)^{\rho}}, \quad 0 \leq T \leq+\infty .
$$

Remark 6. From the above definitions, it can be seen easily that the concepts of $(p, q)$-order and $(p, q)$-type can cover the above shortcomings (S1) and (S2) in some sense.

However, these concepts are inadequate for comparing the growth of LaplaceStieltjes transforms of the same $(p, q)$-orders but of infinite $(p, q)$-types. Inspired by this question, one purpose of this paper is to improve the above inadequacy and investigate the growth of LaplaceStieltjes transforms.

The structure of this paper is listed as following. Section 2 is to investigate the growth of LaplaceStieltjes transforms with the $(p, q)$-proximate order, and an equivalence theorem on the $(p, q)$-proximate order is obtained among the $(p, q)$-order $\rho(p, q),(p, q)$-type $T(p, q), \lambda_{n}$, and $A_{n}^{*}$. Section 3 is to deal with a study of the approximation on LaplaceStieltjes transforms of the $(p, q)$-proximate order and gives a relation theorem about the error $E_{n}(F, \beta),(p, q)$-order $\rho(p, q),(p, q)$-type $T(p, q)$, and $(p, q)$-proximate order. The topic of growth and approximation of LaplaceStieltjes transforms of $(p, q)$-proximate order, it seems that this topic has never been treated before. Our results in this paper are some improvements of the previous theorems given by Kong, Luo, and Sun.

\section{The $(p, q)$-Proximate Order of LaplaceStieltjes Transforms}

Next, we introduce the definition of $(p, q)$-proximate order of LaplaceStieltjes transforms.

Definition 5. A positive function $\rho(\sigma)$ defined on $\left[\sigma_{0},+\infty\right)$, $\sigma_{0}>0$, is a proximate order of LaplaceStieltjes transforms (11) with index-pair $(p, q)$ if $\rho(\sigma)$ satisfies the following conditions:

$$
\begin{aligned}
& \text { (i) } \rho(\sigma) \longrightarrow \rho:=\rho(p, q) \text { as } \sigma \longrightarrow+\infty, b<\rho:=\rho(p, q) \\
&<+\infty \text {, where } b=1 \text { if } p=q \text { and } b=0 \text { if } p>q \text {. } \\
& \text { (ii) } \Lambda_{q}(\sigma) \rho^{\prime}(\sigma) \longrightarrow 0 \text { as } \sigma \longrightarrow+\infty \text {, where } \Lambda_{q}(\sigma)= \\
& \\
& \prod_{i=0}^{q} \log ^{i} \sigma .
\end{aligned}
$$

(iii) if

$$
T^{*}=T^{*}(p, q)=\limsup _{\sigma \longrightarrow+\infty} \frac{\log ^{p} M_{u}(\sigma, F)}{\left(\log ^{q-1} \sigma\right)^{\rho(\sigma)}}, \quad 0 \leq T \leq+\infty,
$$

then $\rho(\sigma)$ is said to be the $(p, q)$-proximate order of the LaplaceStieltjes transform $F(s)$ if $0<T^{*}<+\infty$.

Let

$$
x=\left(\log ^{q-1} \sigma\right)^{\rho(\sigma)-\varrho},
$$

where $\varrho=1$ if $q=1$ and zero otherwise, by a simple computation, we can get that $\left(\log ^{q-1} \sigma\right)^{\rho(\sigma)-\varrho}$ is a monotone increase function of $\sigma$ for $\sigma>\sigma_{0}$. Thus, we can define a real function $\Phi(x)$ of $x>x_{0}$ such that

$$
x=\left(\log ^{q-1} \sigma\right)^{\rho(\sigma)-\varrho} \Longleftrightarrow \Phi(x)=\log ^{q-1} \sigma .
$$

Here, one main theorem of this paper is as follows.

Theorem 2. If the LaplaceStieltjes transform (9) satisfies $\sigma_{u}^{F}=+\infty$ (the sequence $\left\{\lambda_{n}\right\}$ satisfy (3), (6), and (11)) and is of $(p, q)$-order $\rho(0<\rho<\infty)$, then 


$$
T^{*}:=T^{*}(p, q)=\limsup _{\sigma \longrightarrow+\infty} \frac{\log ^{p} M_{u}(\sigma, F)}{\left(\log ^{q-1} \sigma\right)^{\rho(\sigma)}} \Leftrightarrow \limsup _{n \longrightarrow+\infty} H\left[\frac{\Phi\left(\log ^{p-1} \lambda_{n}\right)}{\log ^{q}\left(\left(A_{n}^{*}\right)^{-1 / \lambda_{n}}\right)}\right]^{\rho-\varrho}=T^{*}
$$

where $\varrho=1$ if $q=1$ and zero otherwise, and

$$
H= \begin{cases}\frac{1}{e \rho}, & \text { if }(p, q)=(1,0) \\ \frac{(\rho-1)^{\rho-1}}{\rho^{\rho}}, & \text { if }(p, q)=(1,1) \\ 1, & \text { if } p \geq q \geq 2 .\end{cases}
$$

To prove this theorem, we require the following lemmas.

Lemma 1. For the function $\Phi(x)$ defined in (20) and any positive real number $\kappa(0<\kappa<+\infty)$, we have

$$
\lim _{x \rightarrow+\infty} \frac{\Phi(\kappa x)}{\Phi(x)}=\kappa^{1 /(\rho-\varrho)},
$$

where $\varrho$ is stated as in Theorem 2.

Proof. Here, two cases will be considered as follows.

Case 1. If $q=0$, then $\varrho=0$ and $\Phi(x)=\exp (\sigma)$, i.e., $x=$ $\exp (\sigma \rho(\sigma))$. Thus, it follows that $\sigma \longrightarrow+\infty$ as $x \longrightarrow+\infty$ and $\kappa x=\kappa \exp (\sigma \rho(\sigma))$, and $\Phi(\kappa x)=\kappa^{1 /(\rho(\sigma))} \exp (\sigma)$. Since $\lim _{\sigma \longrightarrow+\infty} \rho(\sigma)=\rho$, it yields

$$
\lim _{x \longrightarrow+\infty} \frac{\Phi(\kappa x)}{\Phi(x)}=\lim _{x \longrightarrow+\infty} \frac{\kappa^{1 /(\rho(\sigma))} \exp (\sigma)}{\exp (\sigma)}=\kappa^{1 /(\rho)} .
$$

Case 2. If $q>0$. From Definition 4 , we conclude

$$
\lim _{x \longrightarrow+\infty} \frac{d[\log \Phi(x)]}{d(\log x)}=\lim _{x \longrightarrow+\infty} \frac{1}{\rho(\sigma)-\varrho+\Lambda_{q}(\sigma) \rho^{\prime}(\sigma)}=\frac{1}{\rho-\varrho} .
$$

Thus, for any given $\varepsilon>0$ and $\kappa>1$, from (28) it follows

$$
\begin{aligned}
\int_{x}^{\kappa x}\left(\frac{1}{\rho-\varrho}-\varepsilon\right) d(\log t) & <\int_{x}^{\kappa x} d(\log \Phi(t)) \\
& <\int_{x}^{\kappa x}\left(\frac{1}{\rho-\varrho}+\varepsilon\right) d(\log t),
\end{aligned}
$$

that is,

$$
\left(\frac{1}{\rho-\varrho}-\varepsilon\right) \log \kappa<\log \frac{\Phi(\kappa x)}{\Phi(x)}<\left(\frac{1}{\rho-\varrho}+\varepsilon\right) \log \kappa
$$

Hence,

$$
\kappa^{1 /(\rho-\varrho)-\varepsilon}<\frac{\Phi(\kappa x)}{\Phi(x)}<\kappa^{1 /(\rho-\varrho)+\varepsilon} .
$$

For $\kappa=1$, it is obviously. For $0<\kappa<1$, by a similar argument as in (28), we can deduce

$$
\begin{aligned}
\int_{\kappa x}^{x}\left(\frac{1}{\rho-\varrho}-\varepsilon\right) d(\log t) & <\int_{\kappa x}^{x} d(\log \Phi(t)) \\
& <\int_{\kappa x}^{x}\left(\frac{1}{\rho-\varrho}+\varepsilon\right) d(\log t),
\end{aligned}
$$

that is,

$$
-\left(\frac{1}{\rho-\varrho}-\varepsilon\right) \log \kappa<\log \frac{\Phi(x)}{\Phi(\kappa x)}<-\left(\frac{1}{\rho-\varrho}+\varepsilon\right) \log \kappa .
$$

Hence,

$$
\kappa^{-(1 /(\rho-\varrho)-\varepsilon)}<\frac{\Phi(x)}{\Phi(\kappa x)}<\kappa^{-(1 /(\rho-\varrho)+\varepsilon)} .
$$

Thus, this completes the proof of Lemma 1 from Cases 1 and 2 .

Lemma 2 (see [10], Lemma 2.1). If the L-S transform $F(s) \in L_{\infty}$, for sufficiently larger $\sigma \longrightarrow+\infty$ and $\varepsilon(>0)$, we have

$$
\frac{1}{2} \mu(\sigma, F) \leq M_{u}(\sigma, F) \leq C \mu((1+2 \varepsilon) \sigma, F)
$$

where $C$ is a constant.

Remark 7. From Lemma 2, we can easily get the following result:

If the LaplaceStieltjes transform (11) satisfies $\sigma_{u}^{F}=+\infty$ (the sequence $\left\{\lambda_{n}\right\}$ satisfy (3), (9), and (20)) and is of $(p, q)$-order $\rho(0<\rho<\infty)$, then

$$
\begin{aligned}
T^{*} & :=T^{*}(p, q)=\underset{\sigma \longrightarrow+\infty}{\lim \sup } \frac{\log ^{p} M_{u}(\sigma, F)}{\left(\log ^{q-1} \sigma\right)^{\rho(\sigma)}} \\
& =\limsup _{\sigma \longrightarrow+\infty} \frac{\log ^{p} \mu(\sigma, F)}{\left(\log ^{q-1} \sigma\right)^{\rho(\sigma)}} .
\end{aligned}
$$

2.1. The Proof of Theorem 2. Here, we first prove the necessity of Theorem 2.

$\Longrightarrow$ : We will divide into two steps to prove the necessity.

Step one. We first prove that

$$
\limsup _{n \longrightarrow+\infty} H\left[\frac{\Phi\left(\log ^{p-1} \lambda_{n}\right)}{\log ^{q}\left(\left(A_{n}^{*}\right)^{-1 / \lambda_{n}}\right)}\right]^{\rho-\varrho} \leq T^{*} .
$$

Since

$$
T^{*}:=T^{*}(p, q)=\limsup _{\sigma \longrightarrow+\infty} \frac{\log ^{p} M_{u}(\sigma, F)}{\left(\log ^{q-1} \sigma\right)^{\rho(\sigma)}}
$$


for any given $\varepsilon>0$ and $\sigma>\sigma_{0}$, it follows

$$
\log ^{p} M_{u}(\sigma, F) \leq\left(T^{*}+\varepsilon\right)\left(\log ^{q-1} \sigma\right)^{\rho(\sigma)} .
$$

Let $B=T^{*}+\varepsilon$, then

$$
\log M_{u}(\sigma, F) \leq \exp ^{p-1}\left\{B\left(\log ^{q-1} \sigma\right)^{\rho(\sigma)}\right\} .
$$

It thus follows from Lemma 2 that

$$
\log A_{n}^{*} \leq \exp ^{p-1}\left\{B\left(\log ^{q-1} \sigma\right)^{\rho(\sigma)}\right\}-\lambda_{n} \sigma+\log 2 .
$$

Now, we will consider three cases as follows.

Case 3. Suppose that $p=1$ and $q=0$, then $\varrho=0$. From (39), it follows

$$
\log A_{n}^{*} \leq B \exp (\sigma \rho(\sigma))-\lambda_{n} \sigma+\log 2 .
$$

Take $\sigma$ such that

$$
\exp (\sigma \rho(\sigma))=\frac{\lambda_{n}}{B \rho},
$$

that is,

$$
e^{\sigma}=\left(\frac{\lambda_{n}}{B \rho}\right)^{1 /(\rho(\sigma))}
$$

In view of (40) and (43), it yields

$$
\begin{aligned}
\log A_{n}^{*} & \leq \frac{\lambda_{n}}{\rho}-\lambda_{n} \sigma+\log 2 \leq-(1+o(1)) \lambda_{n}\left(\sigma-\frac{1}{\rho}\right), \\
\operatorname{or}\left(A_{n}^{*}\right)^{-1 / \lambda_{n}} & \geq \exp \left(-\frac{1}{\rho}(1+o(1))\right)\left(\frac{\lambda_{n}}{B \rho}\right)^{1 /(\rho(\sigma))},
\end{aligned}
$$

that is,

$$
\frac{\left(\lambda_{n}\right)^{1 /(\rho(\sigma))}}{\left(A_{n}^{*}\right)^{-1 / \lambda_{n}}} \leq \frac{1}{\exp (-1 / \rho(1+o(1)))(1 / B \rho)^{1 /(\rho(\sigma))}} .
$$

Since $q=0$, thus $x=\exp (\sigma \rho(\sigma))$, that is, $\Phi(x)=e^{\sigma}$. From (40), we have $\sigma \longrightarrow+\infty$ and $\rho(\sigma) \longrightarrow \rho$ as $n \longrightarrow+\infty$. Hence, from (43), and combining with $\varepsilon$ is arbitrarily, we can deduce

$$
\limsup _{n \longrightarrow+\infty}\left[\frac{\Phi\left(\lambda_{n}\right)}{\left(A_{n}^{*}\right)^{-1 / \lambda_{n}}}\right]^{\rho} \leq T^{*} e \rho .
$$

Case 4. Suppose that $p=1$ and $q=1$, then $\varrho=1$. From (39), it follows

$$
\log A_{n}^{*} \leq B \sigma^{\rho(\sigma)}-\lambda_{n} \sigma+\log 2 .
$$

Take $\sigma$ such that

$$
\sigma^{\rho(\sigma)-1}=\frac{\lambda_{n}}{B \rho}
$$

From (46), it follows $\sigma=\Phi\left(\lambda_{n} / B \rho\right)$. Thus, we can conclude from (45) that

$$
\log A_{n}^{*} \leq-\lambda_{n} \sigma \frac{\rho-1}{\rho}(1+o(1))=-\lambda_{n} \frac{\rho-1}{\rho}(1+o(1)) \Phi\left(\frac{\lambda_{n}}{B \rho}\right),
$$

that is,

$$
\frac{\Phi\left(\lambda_{n}\right)}{-\left(1 / \lambda_{n}\right) \log A_{n}^{*}} \leq \frac{\Phi\left(\lambda_{n}\right)}{(\rho-1 / \rho)(1+o(1)) \Phi\left(\lambda_{n} / B \rho\right)} .
$$

From (46), it yields $\sigma \longrightarrow+\infty$ and $\rho(\sigma) \longrightarrow \rho$ as $n \longrightarrow+\infty$. Hence, from (48), and combining with $\varepsilon$ is arbitrarily, we conclude

$$
\limsup _{n \longrightarrow+\infty}\left[\frac{\Phi\left(\lambda_{n}\right)}{-\left(1 / \lambda_{n}\right) \log A_{n}^{*}}\right]^{\rho-1} \leq T^{*} \frac{\rho^{\rho}}{(\rho-1)^{\rho-1}} .
$$

Case 5. Suppose that $p \geq q \geq 2$, then $\varrho=0$. Take $\sigma$ such that

$$
\left[\log ^{q-1} \sigma\right]^{\rho(\sigma)}=\frac{1}{B} \log ^{p-1} \frac{\lambda_{n}}{\rho},
$$

that is,

$$
\log ^{q-1} \sigma=\Phi\left(\frac{1}{B} \log ^{p-1} \frac{\lambda_{n}}{\rho}\right) .
$$

Substituting (50) into (38), it yields

$$
\log A_{n}^{*} \leq-\lambda_{n}\left(\sigma-\frac{1}{\rho}\right)+\log 2=-\lambda_{n}\left(\sigma-\frac{1}{\rho}\right)(1+o(1)) \text {. }
$$

Thus, it follows

$$
\begin{aligned}
-\frac{1}{\lambda_{n}} \log A_{n}^{*} & \geq\left(\sigma-\frac{1}{\rho}\right)(1+o(1)) \\
& =\left(\exp ^{q-1}\left\{\Phi\left(\frac{1}{B} \log ^{p-1} \frac{\lambda_{n}}{\rho}\right)\right\}-\frac{1}{\rho}\right)(1+o(1)) \\
& \geq \exp ^{q-1}\left\{\Phi\left(\frac{1}{B} \log ^{p-1} \frac{\lambda_{n}}{\rho}\right)\right\}(1+o(1))
\end{aligned}
$$

that is,

$$
\log ^{q-1}\left(-\frac{1}{\lambda_{n}} \log A_{n}^{*}\right) \geq \Phi\left(\frac{1}{B} \log ^{p-1} \frac{\lambda_{n}}{\rho}\right)(1+o(1)) .
$$

In view of (54), we can deduce

$$
\frac{\Phi\left(\log ^{p-1} \lambda_{n}\right)}{\log ^{q-1}\left(-1 / \lambda_{n} \log A_{n}^{*}\right)} \leq \frac{\Phi\left(\log ^{p-1} \lambda_{n}\right)}{\Phi\left(1 / B \log ^{p-1} \lambda_{n} / \rho\right)(1+o(1))} .
$$

Since $\log ^{p-1}\left(\lambda_{n} / \rho\right) \sim \log ^{p-1} \lambda_{n}$ as $n \longrightarrow+\infty$ and $p>1$, by Lemma 1 , let $\varepsilon \longrightarrow 0$ and $n \longrightarrow+\infty$ in (56), and it yields

$$
\limsup _{n \rightarrow+\infty}\left[\frac{\Phi\left(\log ^{p-1} \lambda_{n}\right)}{\log ^{q-1}\left(-\left(1 / \lambda_{n}\right) \log A_{n}^{*}\right)}\right]^{\rho} \leq T^{*}
$$


Therefore, we can conclude from (44), (46), and (56) that

$$
\limsup _{n \longrightarrow+\infty} H\left[\frac{\Phi\left(\log ^{p-1} \lambda_{n}\right)}{\log ^{q-1}\left(-\left(1 / \lambda_{n}\right) \log A_{n}^{*}\right)}\right]^{\rho} \leq T^{*},
$$

where $H$ is stated as in Theorem 2 .

Step Two. Next, we will prove that

$$
\underset{n \longrightarrow+\infty}{\limsup } H\left[\frac{\Phi\left(\log ^{p-1} \lambda_{n}\right)}{\log ^{q-1}\left(-\left(1 / \lambda_{n}\right) \log A_{n}^{*}\right)}\right]^{\rho}<T^{*},
$$

cannot hold. From (58), let $B^{\prime}$ to be a real constant such that $B^{\prime}<T^{*}$ and

$$
\limsup _{n \longrightarrow+\infty} H\left[\frac{\Phi\left(\log ^{p-1} \lambda_{n}\right)}{\log ^{q-1}\left(-\left(1 / \lambda_{n}\right) \log A_{n}^{*}\right)}\right]^{\rho}=B^{\prime}<T^{*} .
$$

Thus, for any small $\varepsilon$ such that $0<\varepsilon<1 / 3\left(T^{*}-B^{\prime}\right)$ and $k_{0}$ such that $k>k_{0}$, it follows

$$
\Phi\left(\log ^{p-1} \lambda_{n_{k}}\right) \leq\left(\frac{B^{\prime}+\varepsilon}{H}\right)^{1 / \rho-\varrho} \log ^{q-1}\left[-\frac{1}{\lambda_{n_{k}}} \log A_{n_{k}}^{*}\right] .
$$

Here, three cases will be considered as follows.

Case 6. Suppose that $p=1$ and $q=0$, then $\varrho=0$ and $H=1 / e \rho$, and (60) becomes

$$
\Phi\left(\lambda_{n_{k}}\right) \leq\left[e \rho\left(B^{\prime}+\varepsilon\right)\right]^{1 / \rho} \exp \left[-\frac{1}{\lambda_{n_{k}}} \log A_{n_{k}}^{*}\right] .
$$

Thus, it follows

$$
A_{n_{k}}^{*} \leq \exp \left\{-\lambda_{n_{k}} \log \left\{\left[e \rho\left(B^{\prime}+\varepsilon\right)\right]^{-(1 / \rho)} \Phi\left(\lambda_{n_{k}}\right)\right\}\right\},
$$

that is,

$$
\begin{aligned}
\log & {\left[A_{n_{k}}^{*} e^{\lambda_{n_{k}} \sigma}\right] } \\
& \leq \sup _{k \geq 0}\left\{-\lambda_{n_{k}} \log \left\{\left[e \rho\left(B^{\prime}+\varepsilon\right)\right]^{-(1 / \rho)} \Phi\left(\lambda_{n_{k}}\right)\right\}+\lambda_{n_{k}} \sigma\right\} .
\end{aligned}
$$

Take $\sigma$ such that

$$
\sigma=\log \Phi\left(\frac{\lambda_{n_{k}}}{\rho\left(B^{\prime}+\varepsilon\right)}\right)
$$

that is,

$$
e^{\sigma \rho(\sigma)}=\frac{\lambda_{n_{k}}}{\rho\left(B^{\prime}+\varepsilon\right)} .
$$

Then, in view of Lemma 2 and (63), we can deduce

$$
\begin{aligned}
\log \left[A_{n_{k}}^{*} e^{\lambda_{n_{k}} \sigma}\right] & \leq \sup _{k \geq 0}\left\{\lambda_{n_{k}} \sigma-\lambda_{n_{k}} \log \left\{\left[e \rho\left(B^{\prime}+\varepsilon\right)\right]^{-(1 / \rho)}\left[\rho\left(B^{\prime}+\varepsilon\right)\right]^{(1 / \rho)}(1+o(1)) e^{\sigma}\right\}\right\} \\
& \leq \sup _{k \geq 0}\left\{\frac{\lambda_{n_{k}}}{\rho}(1+o(1))\right\} \leq\left(B^{\prime}+\varepsilon\right)(1+o(1)) e^{\sigma \rho(\sigma)} \leq\left(B^{\prime}+2 \varepsilon\right) e^{\sigma \rho(\sigma)} .
\end{aligned}
$$
means

Thus, from Lemma 2 and (66) and since $B^{\prime}+3 \varepsilon<T^{*}$, it

$$
T^{*}=\limsup _{\sigma \longrightarrow+\infty} \frac{\log M_{u}(\sigma, F)}{e^{\sigma \rho(\sigma)}} \leq B^{\prime}+3 \varepsilon<T^{*},
$$

a contradiction.

Case 7. Suppose that $p=1$ and $q=1$, then $\varrho=1$ and $H=(\rho-1)^{\rho-1} / \rho^{\rho}$. From $(60)$, it follows

$$
\log \left[A_{n_{k}}^{*} e^{\lambda_{n_{k}} \sigma}\right] \leq \sup _{k \geq 0}\left\{-\lambda_{n_{k}}\left\{\left[\frac{H}{B^{\prime}+\varepsilon}\right]^{1 / \rho-1} \Phi\left(\lambda_{n_{k}}\right)\right\}+\lambda_{n_{k}} \sigma\right\} .
$$

Take $\sigma$ such that

$$
\sigma=\Phi\left(\frac{\lambda_{n_{k}}}{\rho\left(B^{\prime}+\varepsilon\right)}\right)
$$

that is,

$$
\sigma^{\rho(\sigma)-1}=\frac{\lambda_{n_{k}}}{\rho\left(B^{\prime}+\varepsilon\right)} .
$$

Then, in view of Lemma 1 and (68), it follows

$$
\begin{aligned}
\log \left[A_{n_{k}}^{*} e^{\lambda_{n_{k}} \sigma}\right] & \leq \sup _{k \geq 0}\left\{\lambda_{n_{k}} \sigma-\lambda_{n_{k}}\left[(H \rho)^{1 /(\rho-1)} \sigma(1+o(1))\right]\right\} \\
& \leq \sup _{k \geq 0}\left\{\frac{\lambda_{n_{k}} \sigma}{\rho}(1+o(1))\right\} \\
& \leq \sup _{k \geq 0}\left\{\left(B^{\prime}+\varepsilon\right)(1+o(1)) \sigma^{\rho(\sigma)}\right\}
\end{aligned}
$$

Thus, from Lemma 2 and (71) and since $B^{\prime}+3 \varepsilon<T^{*}$, we conclude

$$
T^{*}=\limsup _{\sigma \longrightarrow+\infty} \frac{\log M_{u}(\sigma, F)}{\sigma^{\rho(\sigma)}} \leq B^{\prime}+3 \varepsilon<T^{*},
$$

a contradiction.

Case 8. Suppose that $p \geq q \geq 2$, then $\varrho=0$ and $H=1$. Then, it follows from (60) that

$$
\begin{aligned}
& \log \left[A_{n_{k}}^{*} e^{\lambda_{n_{k}} \sigma}\right] \\
& \quad \leq \sup _{k \geq 0}\left\{\lambda_{n_{k}} \sigma-\lambda_{n_{k}} \exp ^{q-1}\left\{\left[\frac{1}{B^{\prime}+\varepsilon}\right]^{1 / \rho} \Phi\left(\log ^{p-1} \lambda_{n_{k}}\right)\right\}\right\} .
\end{aligned}
$$


Take $\sigma$ such that

$$
\log ^{q-1}(\sigma-1)=\Phi\left(\frac{\log ^{p-1} \lambda_{n_{k}}}{B^{\prime}+\varepsilon}\right)
$$

$$
\lambda_{n_{k}}=\exp ^{p-1}\left\{\left(B^{\prime}+\varepsilon\right)\left[\log ^{q-1}(\sigma-1)\right]^{\rho(\sigma)}\right\} .
$$

Then, from Lemma 1 and (73), it yields

that is,

$$
\begin{aligned}
\log \left[A_{n_{k}}^{*} e^{\lambda_{n_{k}} \sigma}\right] & \leq \sup _{k \geq 0}\left\{\lambda_{n_{k}} \sigma-\lambda_{n_{k}}[(\sigma-1)(1+o(1))]\right\} \leq \sup _{k \geq 0}\left\{\lambda_{n_{k}}(1+o(1))\right\} \\
& \leq \sup _{k \geq 0}\left\{\exp ^{p-1}\left\{\left(B^{\prime}+\varepsilon\right)\left[\log ^{q-1}(\sigma-1)\right]^{\rho(\sigma)}\right\}(1+o(1))\right\} .
\end{aligned}
$$

Thus, from Lemma 2 and (76) and since $B^{\prime}+3 \varepsilon<T^{*}$ and $q \geq 2$, we conclude

$$
T^{*}=\limsup _{\sigma \longrightarrow+\infty} \frac{\log ^{p} M_{u}(\sigma, F)}{\left(\log ^{q-1} \sigma\right)^{\rho(\sigma)}} \leq B^{\prime}+3 \varepsilon<T^{*},
$$

a contradiction.

Therefore, the necessity of Theorem 2 is proved from Steps one and two.

In fact, by using the same argument as in the processing of the necessity of Theorem 2 , we can easily prove the sufficiency of Theorem 2. Hence, this completes the proof of Theorem 2.

\section{The Approximation of LaplaceStieltjes Transforms}

We denote $\bar{L}_{\beta}$ to be a class of all the functions $F(s)$ of form (9) which are analytic in the half plane $\mathfrak{R} s<\beta(-\infty<\beta<\infty)$ and the sequence $\left\{\lambda_{n}\right\}$ satisfy (3) and (6), and denote $L_{\infty}$ to be the class of all the functions $F(s)$ of form (9) which are analytic in the half plane $\mathfrak{R} s<+\infty$ and the sequence $\left\{\lambda_{n}\right\}$ satisfy (3), (6), and (11). Thus, if $-\infty<\beta<+\infty$ and $F(s) \in \bar{L}_{\beta}$, then $F(s) \in L_{\infty}$. If the LaplaceStieltjes transform (11), $A_{n}^{*}=0$ for $n \geq k+1$, and $A_{k}^{*} \neq 0$, then $F(s)$ will be called an exponential polynomial of degree $k$ usually denoted by $p_{k}$, i.e., $p_{k}(s)=\int_{0}^{\lambda_{k}} \exp (s y) \mathrm{d} \alpha(y)$. When we choose a suitable function $\alpha(y)$, the function $p_{k}(s)$ may be reduced to a polynomial in terms of $\exp \left(s \lambda_{i}\right)$, that is, $\sum_{i=1}^{k} b_{i} \exp \left(s \lambda_{i}\right)$.

For $F(s) \in \bar{L}_{\beta}$ and $-\infty<\beta<+\infty$, we denote by $E_{n}(F, \beta)$ the error in approximating the function $F(s)$ by exponential polynomials of degree $n$ in uniform norm as

$$
E_{n}(F, \beta)=\inf _{p \in \Pi_{n}}\|F-p\|_{\beta}, \quad n=1,2, \ldots,
$$

where

$$
\|F-p\|_{\beta}=\max _{-\infty<t<+\infty}|F(\beta+i t)-p(\beta+i t)| .
$$

In 2017, Singhal and Srivastava [28] studied the approximation of LaplaceStieltjes transforms of finite order and obtained the following theorem.

Theorem 3 (see [28]). If the LaplaceStieltjes transform $F(s) \in L_{\infty}$ and is of order $\rho(0<\rho<\infty)$ and of type $T$, then for any real number $-\infty<\beta<+\infty$, we have

$$
\begin{aligned}
\rho & =\limsup _{n \longrightarrow+\infty} \frac{\lambda_{n} \log \lambda_{n}}{-\log E_{n-1}(F, \beta) \exp \left(-\beta \lambda_{n}\right)} \\
& =\limsup _{n \longrightarrow+\infty} \frac{\lambda_{n} \log \lambda_{n}}{-\log E_{n-1}(F, \beta)}, \\
T & =\limsup _{n \longrightarrow+\infty} \frac{\lambda_{n}}{\rho e}\left(E_{n-1}(F, \beta) \exp \left(-\beta \lambda_{n}\right)\right)^{\rho / \lambda_{n}} \\
& =\limsup _{n \longrightarrow+\infty} \frac{\lambda_{n}}{\rho \exp (\rho \beta+1)}\left(E_{n-1}(F, \beta)\right)^{\rho / \lambda_{n}} .
\end{aligned}
$$

Recently, the second author investigated the approximation of LaplaceStieltjes transforms of infinite order by using the concepts of $X$-order and the type function and obtained some theorems as follows.

Theorem 4 (see [29]). If the LaplaceStieltjes transform $F(s) \in L_{0}$, then for any fixed real number $-\infty<\beta<0$, we have

$$
\begin{aligned}
\limsup & \frac{X\left(\log ^{+} M_{u}(\sigma, F)\right)}{\log (-(1 / \sigma))} \\
& =\rho^{*} \Leftrightarrow \limsup _{n \rightarrow \infty} \frac{X\left(\lambda_{n}\right)}{\log ^{+}\left(\lambda_{n} / \log ^{+}\left[E_{n-1}(F, \beta) \exp \left\{-\beta \lambda_{n}\right\}\right]\right)}=\rho^{*},
\end{aligned}
$$

where $0<\rho^{*}<\infty$.

Theorem 5 (see [29]). If the LaplaceStieltjes transform $F(s) \in L_{0}$, then for any fixed real number $-\infty<\beta<0$, we have

$$
\begin{aligned}
\limsup & \frac{X\left(\log ^{+} M_{u}(\sigma, F)\right)}{\log U(-(1 / \sigma))} \\
\quad=1 \Leftrightarrow 0^{-} & \limsup _{n \longrightarrow \infty} \frac{X\left(\lambda_{n}\right)}{\log U\left(\left(\lambda_{n} / \log ^{+}\left[E_{n-1}(F, \beta) \exp \left\{-\beta \lambda_{n}\right\}\right]\right)\right)}=1 .
\end{aligned}
$$

Remark 8 . The definitions of $X$-order and the type function $U(x)$ can be found in $[30,31]$.

The other purpose of this paper is to investigate the approximation of LaplaceStieltjes transforms with 
proximate order $\rho(\sigma)$, the result concerning the error $E_{n}(F, \beta)$, and the proximate order of such function is obtained. The main theorem is listed below.
Theorem 6. If the LaplaceStieltjes transform $F(s) \in L_{\infty}$ and is of $(p, q)$ proximate order $T^{*}\left(0<T^{*}<\infty\right)$, then for any real number $-\infty<\beta<+\infty$, we have

$$
T^{*}= \begin{cases}\limsup _{n \longrightarrow+\infty} H\left[\frac{\Phi\left(\log ^{p-1} \lambda_{n}\right)}{\log ^{q}\left(\left[E_{n-1}(F, \beta)\right]^{-\left(1 / \lambda_{n}\right)}\right)}\right]^{\rho-\varrho}, & \text { if }(p, q)=(1,1) \text { or } q \geq 2 ; \\ \limsup _{n \longrightarrow+\infty} \frac{1}{\rho \exp (\beta \rho+1)}\left[\frac{\Phi\left(\lambda_{n}\right)}{\left[E_{n-1}(F, \beta)\right]^{-\left(1 / \lambda_{n}\right)}}\right]^{\rho}, & \text { if }(p, q)=(1,0) .\end{cases}
$$

where $\rho(\sigma), \varrho$, and $H$ are stated as in Theorem 2.

Proof. Suppose that

$$
T^{*}:=T^{*}(p, q)=\limsup _{\sigma \longrightarrow+\infty} \frac{\log ^{p} M_{u}(\sigma, F)}{\left(\log ^{q-1} \sigma\right)^{\rho(\sigma)}},
$$

then for any given $\varepsilon>0$ and $\sigma>\sigma_{0}$, we have

$$
\log M_{u}(\sigma, F) \leq \exp ^{p-1}\left\{B\left(\log ^{q-1} \sigma\right)^{\rho(\sigma)}\right\},
$$

where $B=T^{*}+\varepsilon$. Since $F(s) \in L_{\infty}$, thus for any constant $\beta(-\infty<\beta<+\infty)$, we have $F(s) \in \bar{L}_{\beta}$. For $\beta<\sigma<+\infty$, it follows from the definitions of $E_{n}(F, \beta)$ and $p_{n}$ that

$$
\begin{aligned}
E_{n}(F, \beta) & \leq\left\|F-p_{n}\right\|_{\beta} \leq\left|F(\beta+i t)-p_{n}(\beta+i t)\right| \\
& \leq\left|\int_{0}^{+\infty} \exp \{(\beta+i t) y\} \mathrm{d} \alpha(y)-\int_{0}^{\lambda_{n}} \exp \{(\beta+i t) y\} \mathrm{d} \alpha(y)\right| \\
& =\left|\int_{\lambda_{n}}^{\infty} \exp \{(\beta+i t) y\} \mathrm{d} \alpha(y)\right| .
\end{aligned}
$$

Set

$$
\begin{aligned}
I(x ; i t) & =\int_{0}^{x} \exp \{i t y\} \mathrm{d} \alpha(y), \\
I_{j+k}(b ; i t) & =\int_{\lambda_{j+k}}^{b} \exp \{i t y\} \mathrm{d} \alpha(y) \quad\left(\lambda_{j+k}<b \leq \lambda_{j+k+1}\right) .
\end{aligned}
$$

Then, we have $\left|I_{j+k}(b ; i t)\right| \leq A_{j+k}^{*}$. Since

$$
\begin{aligned}
& \left|\int_{\lambda_{k}}^{\infty} \exp \{(\beta+i t) y\} \mathrm{d} \alpha(y)\right| \\
& \quad=\lim _{b \longrightarrow+\infty}\left|\int_{\lambda_{k}}^{b} \exp \{(\beta+i t) y\} \mathrm{d} \alpha(y)\right|,
\end{aligned}
$$

where $\beta$ is an any real number, it follows

$$
\begin{aligned}
\left|\int_{\lambda_{k}}^{b} \exp \{(\beta+i t) y\} \mathrm{d} \alpha(y)\right| \\
=\left|\sum_{j=k}^{n+k-1} \int_{\lambda_{j}}^{\lambda_{j+1}} \exp \{\beta y\} d_{y} I_{j}(y ; i t)+\int_{\lambda_{n+k}}^{b} \exp \{\beta y\} d_{y} I_{n+k}(y ; i t)\right| \\
=\mid\left[\sum_{j=k}^{n+k-1} e^{\lambda_{j+1} \beta} I_{j}\left(\lambda_{j+1} ; i t\right)-\beta \int_{\lambda_{j}}^{\lambda_{j+1}} e^{\beta y} I_{j}(y ; i t) \mathrm{d} y\right] \\
\quad+e^{\beta b} I_{n+k}(b ; i t)-\beta \int_{\lambda_{n+k}}^{b} e^{\beta y} I_{j}(y ; i t) \mathrm{d} y \mid \\
\quad \leq \sum_{j=k}^{n+k-1}\left[A_{j}^{*} e^{\lambda_{j+1} \beta}+A_{j}^{*}\left(e^{\lambda_{j+1} \beta}-e^{\lambda_{j} \beta}\right)\right]+2 e^{\beta \lambda_{n+k+1}} A_{n+k}^{*}-e^{\beta \lambda_{n+k}} A_{n+k}^{*} \\
\leq 2 \sum_{j=k}^{n+k} A_{n}^{*} e^{\lambda_{n+1} \beta} .
\end{aligned}
$$


When $n \longrightarrow+\infty$, we have $b \longrightarrow+\infty$. It yields that

$$
\left|\int_{\lambda_{k}}^{\infty} \exp \{(\beta+i t) y\} \mathrm{d} \alpha(y)\right| \leq 2 \sum_{n=k}^{+\infty} A_{n}^{*} \exp \left\{\beta \lambda_{n+1}\right\} .
$$

Thus, from the definition of $A_{n}^{*}$ and $M_{u}(\sigma, F)$ and by Lemma 2, we have $A_{n}^{*} \leq 2 M_{u}(\sigma, F) e^{-\sigma \lambda_{n}}$ for any $\sigma(\beta<\sigma<+\infty)$, it follows from (86) and (90) that

$$
\begin{aligned}
E_{n}(F, \beta) & \leq 2 \sum_{k=n+1}^{\infty} A_{k-1}^{*} \exp \left\{\beta \lambda_{k}\right\} \\
& \leq 4 M_{u}(\sigma, F) \sum_{k=n+1}^{\infty} \exp \left\{(\beta-\sigma) \lambda_{k}\right\} .
\end{aligned}
$$

From (6), take $h^{\prime}\left(0<h^{\prime}<h\right)$ such that $\left(\lambda_{n+1}-\lambda_{n}\right) \geq h^{\prime}$ for $n \geq 0$.Then, for $\sigma \geq \beta+1$, it follows from (91) that that is,

$$
E_{n-1}(F, \beta) \leq K M_{u}(\sigma, F) \exp \left\{\lambda_{n}(\beta-\sigma)\right\},
$$

where $K$ is a constant. Thus, from (85) and (93), it yields that

$$
\begin{aligned}
& \log \left[E_{n-1}(F, \beta) \exp \left(-\beta \lambda_{n}\right)\right] \\
& \quad \leq \exp ^{p-1}\left\{B\left(\log ^{q-1} \sigma\right)^{\rho(\sigma)}\right\}-\lambda_{n} \sigma+\log K,
\end{aligned}
$$

where $\varepsilon>0$ and $\sigma>\sigma_{0}$.

Next, three cases will be considered.

Case 9. Suppose that $p=1$ and $q=0$, then $\varrho=0$ and $H=1 / e \rho$. From (92), it follows

$$
\log \left[E_{n-1}(F, \beta) \exp \left(-\beta \lambda_{n}\right)\right] \leq B e^{\sigma \rho(\sigma)}-\lambda_{n} \sigma+\log K .
$$

Taking $\sigma$ such that

$$
\exp (\sigma)=\Phi\left(\frac{\lambda_{n}}{B \rho}\right)
$$

by using the same argument as in Case 3, we can easily get

$$
\limsup _{n \longrightarrow+\infty}\left[\frac{\Phi\left(\lambda_{n}\right)}{\left[E_{n-1}(F, \beta) \exp \left(-\beta \lambda_{n}\right)\right]^{-\left(1 / \lambda_{n}\right)}}\right]^{\rho} \leq T^{*} e \rho,
$$

that is,

$$
\limsup _{n \longrightarrow+\infty} \frac{1}{\rho \exp (\beta \rho+1)}\left\{\frac{\Phi\left(\lambda_{n}\right)}{\left[E_{n-1}(F, \beta)\right]^{-\left(1 / \lambda_{n}\right)}}\right\}^{\rho} \leq T^{*} .
$$

Now, we need to prove that

$$
\limsup _{n \rightarrow+\infty} \frac{1}{\rho \exp (\beta \rho+1)}\left\{\frac{\Phi\left(\lambda_{n}\right)}{\left[E_{n-1}(F, \beta)\right]^{-\left(1 / \lambda_{n}\right)}}\right\}^{\rho}<T^{*},
$$

does not hold. Let $B^{\prime}$ to be a real constant such that $B^{\prime}<T^{*}$ and
Since for any $\beta<+\infty$, from the definition of $E_{k}(F, \beta)$, there exists $p_{1} \in \Pi_{n-1}$ such that

$$
\left\|F-p_{1}\right\| \leq 2 E_{n-1}(F, \beta) .
$$

And since

$$
\begin{aligned}
A_{n}^{*} \exp \left\{\beta \lambda_{n}\right\} & =\sup _{\lambda_{n}<x \leq \lambda_{n+1},-\infty<t<+\infty}\left|\int_{\lambda_{n}}^{x} \exp \{i t y\} \mathrm{d} \alpha(y)\right| \exp \left\{\beta \lambda_{n}\right\} \\
& \leq \sup _{\lambda_{n}<x \leq \lambda_{n+1},-\infty<t<+\infty}\left|\int_{\lambda_{n}}^{x} \exp \{(\beta+i t) y\} \mathrm{d} \alpha(y)\right| \\
& \leq \sup _{-\infty<t<+\infty}\left|\int_{\lambda_{n}}^{\infty} \exp \{(\beta+i t) y\} \mathrm{d} \alpha(y)\right|
\end{aligned}
$$

thus for any $p \in \Pi_{n-1}$, it means that

$$
A_{n}^{*} \exp \left\{\beta \lambda_{n}\right\} \leq|F(\beta+i t)-p(\beta+i t)| \leq\|F-p\|_{\beta} .
$$

Hence, from (103) and (104), for any $\beta<+\infty$ and $F(s) \in L_{\infty}$, we can deduce

$$
A_{n}^{*} \exp \left\{\beta \lambda_{n}\right\} \leq 2 E_{n-1}(F, \beta) .
$$

From (101) and (105), it follows

$$
\begin{aligned}
\log & {\left[A_{n_{k}}^{*} e^{\lambda_{n_{k}} \sigma}\right] } \\
& \leq \sup _{k \geq 0}\left\{-\lambda_{n_{k}} \log \left\{\left[e \rho\left(B^{\prime}+\varepsilon\right)\right]^{-(1 / \rho)} \Phi\left(\lambda_{n_{k}}\right)\right\}+\lambda_{n_{k}} \sigma+\log 2\right\} .
\end{aligned}
$$


Taking $\sigma_{k}$ such that

$$
\sigma_{k}=\log \Phi\left(\frac{\lambda_{n_{k}}}{\rho\left(B^{\prime}+\varepsilon\right)}\right)
$$

by using the same argument as in Case 6, we have

$$
T^{*}=\limsup _{\sigma \longrightarrow+\infty} \frac{\log M_{u}(\sigma, F)}{e^{\sigma \rho(\sigma)}} \leq B^{\prime}+3 \varepsilon<T^{*},
$$

a contradiction.

Case 10. Suppose that $p=1$ and $q=1$; then, $\varrho=1$ and $H=(\rho-1)^{\rho-1} / \rho^{\rho}$. Then, it follows from (101) that

$$
\log \left[E_{n-1}(F, \beta) \exp \left(-\beta \lambda_{n}\right)\right] \leq B \sigma^{\rho(\sigma)}-\lambda_{n} \sigma+\log K .
$$

Taking $\sigma$ such that

$$
\sigma=\Phi\left(\frac{\lambda_{n}}{B \rho}\right)
$$

by using the same argument as in Case 3, we can easily get

$$
\limsup _{n \longrightarrow+\infty} H\left[\frac{\Phi\left(\lambda_{n}\right)}{-\left(1 / \lambda_{n}\right) \log \left[E_{n-1}(F, \beta) \exp \left(-\beta \lambda_{n}\right)\right]}\right]^{\rho-1} \leq T^{*} .
$$

If

$$
\limsup _{n \longrightarrow+\infty} H\left[\frac{\Phi\left(\lambda_{n}\right)}{-\left(1 / \lambda_{n}\right) \log \left[E_{n-1}(F, \beta) \exp \left(-\beta \lambda_{n}\right)\right]}\right]^{\rho-1}<T^{*},
$$

by combining the proof of Case 7 and Case 9, a contradiction can be easily obtained.

Case 11. Suppose that $p \geq q \geq 2$, then $\varrho=0$ and $H=1$. Take $\sigma$ such that

$$
\log ^{q-1} \sigma=\Phi\left(\frac{1}{B} \log ^{p-1} \frac{\lambda_{n}}{\rho}\right)
$$

By using the same argument as in Case 5, we can conclude from (94) that

$$
\limsup _{n \longrightarrow+\infty}\left[\frac{\Phi\left(\log ^{p-1} \lambda_{n}\right)}{\log ^{q-1}\left(-\left(1 / \lambda_{n}\right) \log \left[E_{n-1}(F, \beta) \exp \left(-\beta \lambda_{n}\right)\right]\right)}\right]^{\rho} \leq T^{*}
$$

$$
\limsup _{n \rightarrow+\infty}\left[\frac{\Phi\left(\log ^{p-1} \lambda_{n}\right)}{\log ^{q-1}\left(-\left(1 / \lambda_{n}\right) \log \left[E_{n-1}(F, \beta) \exp \left(-\beta \lambda_{n}\right)\right]\right)}\right]^{\rho}<T^{*},
$$

by combining the proof of Case 8 and Case 9 , a contradiction can be easily obtained.

Therefore, this completes the proof of Theorem 6 .

\section{Data Availability}

No data were used to support this study.

\section{Conflicts of Interest}

The authors declare that none of the authors have any conflicts of interest in the manuscript.

\section{Authors' Contributions}

HYX and YQC completed the main part of this article, and HYX corrected the main theorems. All the authors read and approved the final manuscript.

\section{Acknowledgments}

This work was supported by the National Natural Science Foundation of China (11561033), the Natural Science Foundation of Jiangxi Province in China (20181BAB201001), and the Foundation of Education Department of Jiangxi (GJJ190876, GJJ190895, and GJJ191042) of China.

\section{References}

[1] J. R. Yu, "Borel's line of entire functions represented by Laplace-Stieltjes transformation," Acta Mathematica Sinica, vol. 13, pp. 471-484, 1963, in Chinese.

[2] C. J. K. Batty, "Tauberian theorems for the Laplace-Stieltjes transform," Transactions of the American Mathematical Society, vol. 322, no. 2, pp. 783-804, 1990.

[3] X. Chang, S. Liu, P. Zhao, and D. Song"A generalization of linearized alternating direction method of multipliers for solving two-block separable convex programming," Journal of Computational and Applied Mathematics, vol. 357, pp. 251-272, 2019.

[4] X. Chang, S. Liu, P. Zhao, and X. Li, "Convergent predictioncorrection-based ADMM for multi-block separable convex programming," Journal of Computational and Applied Mathematics, vol. 335, pp. 270-288, 2018.

[5] K. Knopp, "Über die Konvergenzabszisse des Laplace-Integrals," Mathematische Zeitschrift, vol. 54, pp. 291-296, 1951.

[6] A. Mishkelyavichyus, "A Tauberian theorem for the LaplaceStieltjes integral and the Dirichlet series (Russian)," Lithuanian Mathematical Journal, vol. 29, no. 4, pp. 364-369, 1990.

[7] O. Posiko and M. Sheremeta, "Logarithms of Laplace-Stiltjes integral and maximum of the integrand," Integral Transforms and Special Functions, vol. 18, no. 4, pp. 271-283, 2007.

[8] M. Ru, "The recent progress in Nevanlinna theory," Journal of Jiangxi Normal University. Natural Sciences Edition, vol. 42, pp. 1-11, 2018.

[9] L. N. Shang and Z. S. Gao, "The growth of entire functions of infinite order represented by Laplace-Stieltjes transformation," Acta Mathematica Scientia, vol. 27A, no. 6, pp. 10351043, 2007, in Chinese.

[10] L. N. Shang and Z. S. Gao, "The value distribution of analytic functions defined by Laplace-Stieltjes transforms," Acta Mathematica Scientia, vol. 51, no. 5, pp. 993-1000, 2008, in Chinese.

[11] M. M. Sheremeta, "Asymptotical behaviour of Laplace-Stiltjes integrals," in Mathematical Studies, VNTL Publishers, Lviv, Ukraine, 2010.

[12] H. Y. Xu and S. Y. Liu, "The approximation of LaplaceStieltjes transforms with finite order," Journal of Inequalities and Applications, vol. 164, pp. 1-14, 2017.

[13] H. Y. Xu, S. Y. Liu, and Q. P. Li, "Entire solutions for several systems of nonlinear difference and partial differential-difference equations of Fermat-type," Journal of Mathematical Analysis and Applications, vol. 483, no. 2, Article ID 123641, 2020. 
[14] H. Y. Xu, Y. M. Li, and S. Liu, "The partially shared values and small functions for meromorphic functions in a k-punctured complex plane," Journal of Inequalities and Applications, vol. 2019, Article ID 289, 2019.

[15] H. Y. Xu and Y. Y. Kong, "The approximation of LaplaceStieltjes transformations with finite order on the left half plane," Comptes Rendus Mathematique, vol. 356, no. 1, pp. 63-76, 2018.

[16] R. Ying and H. Y. Xu, "The growth of Hadamard product of random Dirichlet series," Jiangxi Normal University (Natural Science), vol. 43, pp. 513-517, 2019.

[17] X. Luo and Y. Y. Kong, "On the order and type of LaplaceStieltjes transforms of slow growth," Acta Mathematica Sinica, vol. 32A, pp. 601-607, 2012.

[18] G. Valiron, Lectures on the General Theory of Integral Functions, Read Books, Vancouver, Canada, 1992.

[19] Y. Y. Kong, "Laplace-Stieltjes transforms of infinite order in the right half-plane," Acta Mathematica Sinica, vol. 55, pp. 141-148, 2012.

[20] D. Sato, "On the rate of growth of entire functions of fast growth," Bulletin of the American Mathematical Society, vol. 69, no. 3, pp. 411-415, 1963.

[21] K. Nandan, R. P. Doherey, and R. S. L. Srivastava, "On the generalized type and generalized lower type of an entire function with index pair $(p, q)$," Indian Journal of Pure and Applied Mathematics, vol. 11, pp. 1424-1433, 1980.

[22] A. Nautiyal and G. P. Kapoor, "On the generalized orders of an entire function of slow growth," Indian Journal of Pure and Applied Mathematics, vol. 13, pp. 1246-1252, 1982.

[23] G. S. Srivastava, "Degree of convergence for entire functions of slow growth," Matematički Vesnik, vol. 35, pp. 319-330, 1983.

[24] S. K. Vaish and G. S. Srivastava, "On the logarithmic order of entire Dirichlet series," Indian Journal of Pure and Applied Mathematics, vol. 16, pp. 49-54, 1985.

[25] S. K. Bajpai, G. P. Kapoor, and O. P. Juneja, "On entire functions of fast growth," Transactions of the American Mathematical Society, vol. 203, p. 275, 1975.

[26] O. P. Juneja, G. P. Kapor, and S. K. Bajpai, "On the $(p, q)$-order and lower $(p, q)$-order of an entire function," Journal für die Reine und Angewandte Mathematik, vol. 282, pp. 53-67, 1976.

[27] O. P. Juneja, G. P. Kapor, and S. K. Bajpai, "On the $(p, q)$-type and lower $(p, q)$-type of an entire function," Journal für die Reine und Angewandte Mathematik, vol. 290, pp. 180-190, 1977.

[28] C. Singhal and G. S. Srivastava, "On the growth and approximation of entire functions represented by LaplaceStieltjes' transformation," Annali Dell' Universita di Ferrara, vol. 63, no. 2, pp. 365-376, 2017.

[29] H. Y. Xu and Y. Y. Kong, "The approximation of analytic function defined by Laplace-Stieltjes transformations convergent in the left half-plane," Houston Journal of Mathematics, vol. 44, pp. 1121-1134, 2018.

[30] H. Y. Xu and Z. X. Xuan, "The growth and value distribution of Laplace-Stieltjes transformations with infinite order in the right half plane," Journal of Inequalities and Applications, vol. 2013, Article ID 273, 2013.

[31] H. Y. Xu and Z. X. Xuan, "The singular points of analytic functions with finite $\mathrm{X}$-order defined by Laplace-Stieltjes transformations," Journal of Function Spaces, vol. 2015, Article ID 865069, 9 pages, 2015. 